\title{
Identification of Dynamic Systems with Position Dependent Load Parameters
}

\author{
Foeke Vanbecelaere $^{1,3}$, Stijn Derammelaere ${ }^{2,3}$, Jos Knockaert ${ }^{1}$, Kurt Stockman ${ }^{1,3}$, Michael Monte Kr,3 $^{1,3}$
}

\begin{abstract}
Cascaded control is still the most common and convenient structure in standard commercial drives. The most convenient tuning method is to apply calculation rules on an offline identified frequency response of the open-loop system. Obviously, this standard approach is only valid for linear time-invariant systems. However, the mechanical dynamics of modern machines very often depend on the angular position of the driven axis. Consequently, the system is time-variant and linearisation is needed to obtain an open-loop frequency response. In this paper, a system identification approach based on this linearisation is presented for dynamic systems with variable load torque and variable load inertia. The feasibility of this approach is validated with measurements on an industrial case.
\end{abstract}

Index Terms-Closed-loop identification, Motion control, Inertia variation

\section{INTRODUCTION}

Planar mechanisms have the advantage that a rotational movement of the driven joint can be converted to any desired movement of the follower linkage without synchronisation issues. Due to that, modern machines performing repetitive tasks very often use such mechanisms. A few commonly employed examples are the slider-crank mechanism, the four-bar mechanism and the cam mechanism. However, an inherent property is that the reduced moment of inertia at the driven axis is variable [1]. For that reason, the mechanisms are time-variant systems. If such systems are required to move at high-speed, the inertia variation is a challenging parameter for controller tuning [2] and motor selection [3]. The tuning of drives with cascade control is mostly based on an offline identified frequency response of the openloop system. Calculation rules [4] are then applied or tools [5] are used to obtain a desired closed-loop bandwidth. This straight-forward technique requires that the identified open-loop system is reliable. Offline methods to identify the frequency response are well-known. In [6], [7] and [8] torque excitation with a PRBS-signal is proven to be a successful technique for linear systems. In commercial drive manuals (e.g. [9]), methods are described to eliminate nonlinear properties, e.g. static friction, during the identification procedure. Nevertheless, those methods among others are not able to consider the varying dynamics inherent to planar mechanisms.

*This work is supported by the Interreg 2 seas INCASE program, https: / / www. incase2seas.eu/

${ }^{1}$ Department of Electrical Energy, Metals, Mechanical Constructions and Systems, Ghent University Campus Kortrijk, 8500 Kortrijk, Belgium

${ }^{2}$ Department of Electromechanics, Op3Mech, University of Antwerp, 2020 Antwerp, Belgium

${ }^{3}$ Member of EEDT partner of Flanders Make
In this paper a system identification method is proposed

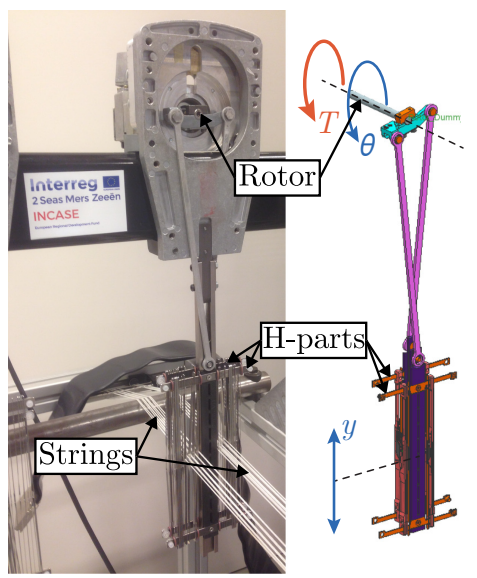

Fig. 1. The physical machine (left) and its CAD equivalent (right)

that can deal with these varying dynamics. An industrial case, shown in figure 1 , is used as a proof of concept. The mechanism is a subsystem of a weaving machine. The system is driven at the rotor with a torque $T$. The rotary movement $\theta$ at the rotor is converted in a vertical movement $y$ of the $\mathrm{H}$-parts by a planar mechanism. Through this movement of the H-parts, rotary split of the strings is achieved. This rotary split is a high-speed repetitive task of $208 \mathrm{~ms}$ per cycle.

The paper is structured as follows. In section II, the weaving unit is represented as a two-mass system and the timevariant motion equations are discussed. Next, in section III the linear system behaviour in the frequency domain is obtained. Thereafter, section IV clarifies the measurement procedure for identification of the time-variant system in a selected operating point where linear system behaviour is valid. Also, important tuning settings for a proper result are discussed. Finally, the experimental identification approach is implemented on the physical machine and the results are presented in section $\mathrm{V}$.

\section{SYSTEM DEFINITION}

For controller tuning, representing a physical machine as a two-mass system is a useful simplification [10]. That way, the most dominant flexibility of the mechanism is represented with a spring-damper connection between rotor and load. In the frequency domain, this assumption results in one pair of antiresonant and resonant peaks and controller tuning is possible. A graphical representation of this model is shown in figure 2. In the motion equations (1) of the two-mass system, $J_{\mathrm{r}}$ is the rotor inertia and $J_{1}$ is the variable load 


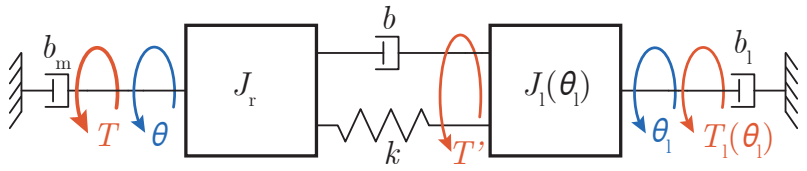

Fig. 2. Model of the two-mass system with variable inertia

inertia depending on the load position $\theta_{1}$. The load inertia is assumed to be the equivalent of all rigidly connected linkages at the load side. The parameters of the coupling are the stiffness $k$ and the damping $b$. The external damping on motor and load side is respectively represented with $b_{\mathrm{m}}$ and $b_{1} . T_{1}$ represents the load torque. In this case the load torque originates from the gravity forces acting on the moving linkages and is thus position dependent. The input of the system is the motor torque $T$ and the output of the system is the rotor speed $\dot{\theta}$.

$$
\left\{\begin{array}{c}
T-b_{\mathrm{m}} \dot{\theta}-b\left(\dot{\theta}-\dot{\theta}_{\mathrm{l}}\right)-k\left(\theta-\theta_{\mathrm{l}}\right)=J_{\mathrm{r}} \ddot{\theta} \\
T_{1}-b_{1} \dot{\theta}_{\mathrm{l}}+b\left(\dot{\theta}-\dot{\theta}_{\mathrm{l}}\right)+k\left(\theta-\theta_{\mathrm{l}}\right)=\frac{1}{2} \dot{J}_{1} \dot{\theta}_{\mathrm{l}}+J_{1} \ddot{\theta}_{\mathrm{l}}
\end{array}\right.
$$

Realistic values of the constant mechanical parameters are given in Table I. The position dependent inertia and load torque are given in figure 3 and are obtained from motion simulations [11] on the CAD equivalent of the physical machine, shown in figure 1 . Table II shows the variable load parameters at three selected positions in figure 3, namely the positions $\theta_{\mathrm{a}}, \theta_{\mathrm{b}}$ and $\theta_{\mathrm{c}}$ of respectively high, medium and low inertia.

Notice that the motion equation (1) of the load side includes
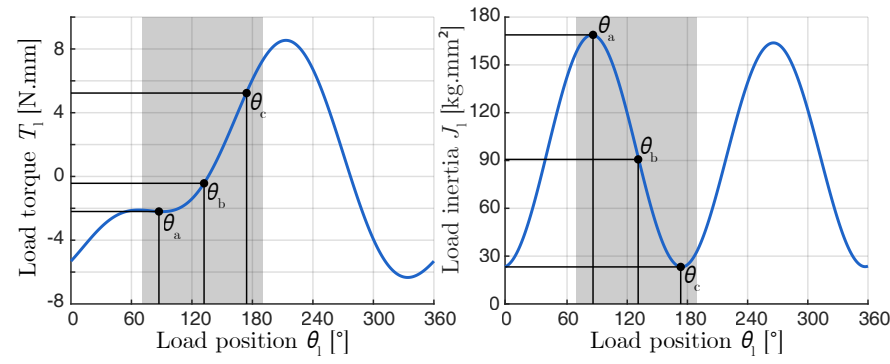

Fig. 3. Variable load torque $T_{1}$ and variable load inertia $J_{1}$

TABLE I

MECHANICAL PARAMETERS OF THE TWO-MASS SYSTEM

\begin{tabular}{|c|c|}
\hline$J_{\mathrm{r}}$ & $28 \mathrm{~kg} \cdot \mathrm{mm}^{2}$ \\
\hline$k$ & $40 \mathrm{Nm} / \mathrm{rad}$ \\
\hline$b$ & $0.01 \mathrm{Nm} \cdot \mathrm{s} / \mathrm{rad}$ \\
\hline$b_{\mathrm{m}}$ & $0.002 \mathrm{Nm} \cdot \mathrm{s} / \mathrm{rad}$ \\
\hline$b_{\mathrm{l}}$ & $0.004 \mathrm{Nm} \cdot \mathrm{s} / \mathrm{rad}$ \\
\hline
\end{tabular}

the time-derivative of the load inertia $\dot{J}_{1}$ (see appendix for the proof of this term). Due to this term and the positiondependent load parameters $T_{1}\left(\theta_{1}\right)$ and $J_{1}\left(\theta_{1}\right)$, the two-mass system depicted in figure 2 is a time-variant system.

\section{LINEARISED SYSTEM DEFINITION}

Linearisation of (1) is needed in order to use the Laplace transform and analyse the system in the frequency domain.
TABLE II

INERTIA AND LOAD TORQUE IN SELECTED POSITIONS

\begin{tabular}{|c|c|c|c|}
\hline & $\theta_{1}\left[{ }^{\circ}\right]$ & $T_{1}[\mathrm{~N} \cdot \mathrm{mm}]$ & $J_{1}\left[\mathrm{~kg} \cdot \mathrm{mm}^{2}\right]$ \\
\hline$\theta_{\mathrm{a}}$ & 86 & -2.20 & 168.85 \\
\hline$\theta_{\mathrm{b}}$ & 131 & -0.53 & 94.78 \\
\hline$\theta_{\mathrm{c}}$ & 175 & 5.40 & 23.18 \\
\hline
\end{tabular}

In a chosen operating point $\theta_{1}=\theta^{*}$, the load inertia $J_{1}\left(\theta^{*}\right)$ is a constant value and thus $\dot{J}_{1}^{*}=0$. The load torque $T_{1}\left(\theta^{*}\right)$ is also constant in that operating point. Hence, the linearised motion equations are obtained:

$$
\left\{\begin{array}{l}
T-b_{\mathrm{m}} \dot{\theta}-b\left(\dot{\theta}-\dot{\theta}_{\mathrm{l}}\right)-k\left(\theta-\theta_{\mathrm{l}}\right)=J_{\mathrm{r}} \ddot{\theta} \\
T_{1}-b_{1} \dot{\theta}_{\mathrm{l}}+b\left(\dot{\theta}-\dot{\theta}_{\mathrm{l}}\right)+k\left(\theta-\theta_{\mathrm{l}}\right)=J_{\mathrm{l}} \ddot{\theta}_{\mathrm{l}}
\end{array}\right.
$$

After Laplace transformation of (2) and using Cramer's rule, the input-output relation is found:

$$
\dot{\theta}(s)=G_{1}(s) T(s)+G_{2}(s) T_{1}(s)
$$

Note that in (3) the system is described with two transfer functions $G_{1}(s)$ (4) and $G_{2}(s)$ (5) that can not be coupled. However, two interesting similarities between $G_{1}(s)$ and $G_{2}(s)$ enable simplification of the linear system behaviour. First of all, the static gain (substitute $s=0$ in (4) and (5)) of both transfer functions is the same.

$$
G_{1}(s=0)=G_{2}(s=0)=\frac{1}{\left(b_{\mathrm{m}}+b_{1}\right)}
$$

Secondly, the pole-zero map in figure 4 shows that both transfer function have the same poles but different zeros. Though, the zero $z_{2}$ is located 5 times further from the

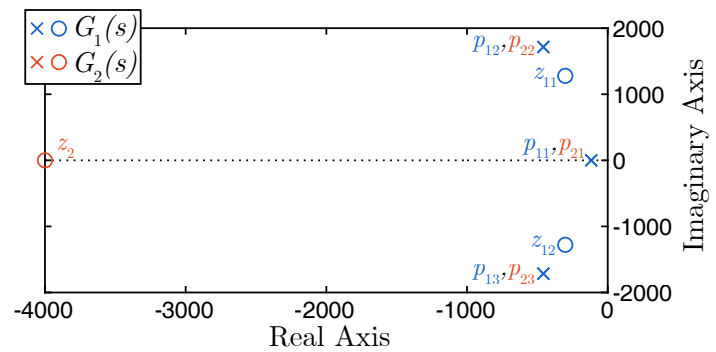

Fig. 4. pole-zero map of $G_{1}(s)$ and $G_{2}(s)$ in the position $\theta_{\mathrm{c}}$ of low inertia

imaginary axis than the dominant poles $p_{21}, p_{22}$ and $p_{23}$. Therefore the influence of $z_{2}$ on the dynamic response of $G_{2}(s)$ is negligible. In conclusion, the input-output relation is fully described by the dynamic response of $G_{1}(s)$ combined with an offset. The offset results from the static gain of $G_{2}(s)$, found in (6), multiplied by the load torque $T_{1}\left(\theta^{*}\right)$ in a selected operating point.

$$
\dot{\theta}(s)=G_{1}(s) T(s)+\frac{T_{1}\left(\theta^{*}\right)}{\left(b_{\mathrm{m}}+b_{1}\right)}
$$

Due to the principle of superposition of the offset in (7), the linear system behaviour is finally described as:

$$
\frac{\dot{\theta}(s)}{T(s)}=G_{1}(s)
$$




$$
\begin{aligned}
& G_{1}(s)=\frac{J_{1} s^{2}+\left(b+b_{1}\right) s+k}{J_{\mathrm{r}} J_{1} s^{3}+\left(\left(J_{\mathrm{r}}+J_{1}\right) b+J_{\mathrm{r}} b_{1}+J_{1} b_{\mathrm{m}}\right) s^{2}+\left(\left(J_{\mathrm{r}}+J_{1}\right) k+b_{\mathrm{m}} b_{1}+\left(b_{\mathrm{m}}+b_{1}\right) b\right) s+\left(b_{\mathrm{m}}+b_{1}\right) k} \\
& G_{2}(s)=\frac{b s+k}{J_{\mathrm{r}} J_{1} s^{3}+\left(\left(J_{\mathrm{r}}+J_{1}\right) b+J_{\mathrm{r}} b_{1}+J_{1} b_{\mathrm{m}}\right) s^{2}+\left(\left(J_{\mathrm{r}}+J_{1}\right) k+b_{\mathrm{m}} b_{1}+\left(b_{\mathrm{m}}+b_{1}\right) b\right) s+\left(b_{\mathrm{m}}+b_{1}\right) k}
\end{aligned}
$$

\section{IDENTIFICATION APPROACH}

The approach to identify the frequency response consists of two parts, namely a measurement part and a dataprocessing part. The setup for the measurement part is shown in figure 5. In light grey, the system to be identified is depicted and the implementation according to its time-variant motion equations (1) is shown in figure 6. The constant mechanical parameters of the system are given in orange and the position-dependent parameters $J_{1}$ and $T_{1}$ are implemented as look-up tables.

During the measurement, it is crucial that the linear system

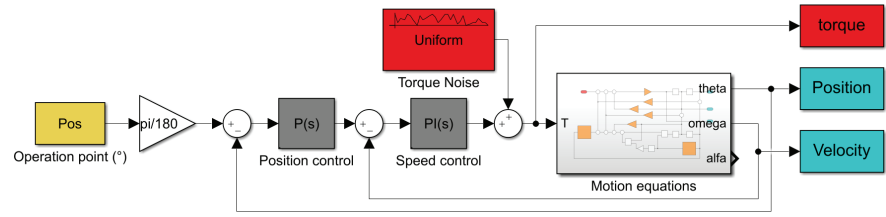

Fig. 5. Simulink implementation of the measurement setup, with the system represented in figure 6

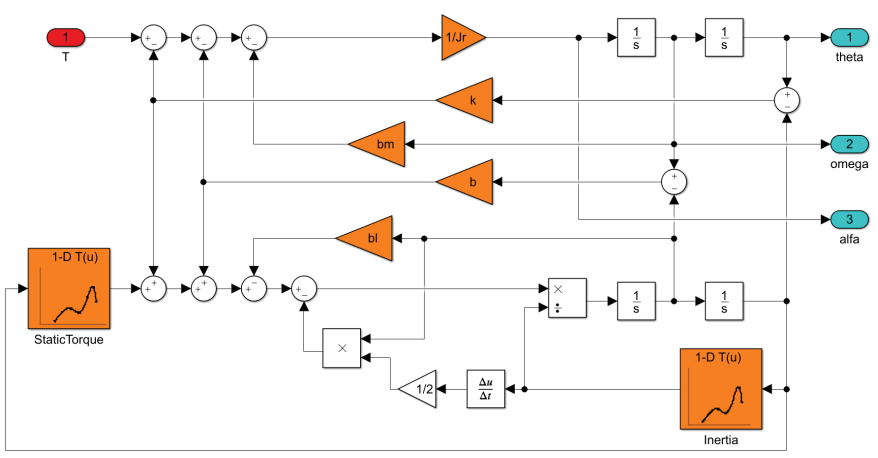

Fig. 6. Simulink implementation of the motion equations (1)

behaviour according to (8) is maintained. As mentioned, this is only possible by keeping the system in the desired operating point $\theta=\theta^{*}$. This is achieved by using a cascade controller (depicted in dark grey) with the operating point $\theta^{*}$ as a set-point (depicted in yellow). For convenience, the structure of the controller is identical to the standard structure in commercial drive software, and consists of a P-controller for the position loop and a PI-controller for the speed loop:

$$
P(s)=K_{\mathrm{p}} \quad P I(s)=K_{\mathrm{i}}\left(1+\frac{1}{T_{\mathrm{i}} s}\right)
$$

Without frequency-rich signals of the system, the frequency response can obviously not be identified. In order to obtain a frequency-rich input signal, a noise generator (depicted in red) is added to the input torque.
To ensure proper performance, a few settings need to be considered. The controller settings are tuned with the objective to have a high robustness and slow response time. That way, the oscillations originating from the noise generator are allowed, while still maintaining an average position of $\theta=\theta^{*}$ and thus maintaining the linear system behaviour. A wide range of controller settings are valid and therefore the tuning is not discussed.

The amplitude $A$ of the noise generator is tuned with the objective to obtain position oscillations with an amplitude of $2^{\circ}-10^{\circ}$ around the desired position $\theta^{*}$. This guideline is found experimentally by trial and error and is later justified with results.

The desired frequency spectrum results from the measurement time $t_{\mathrm{m}}$ and sample time $t_{\mathrm{s}}$. The minimum frequency $f_{\min }$, frequency resolution $\Delta f$ and maximum frequency $f_{\max }$ are found with (10) and are based on the Shannon-Nyquist theorem:

$$
f_{\max }=\frac{1}{2 t_{\mathrm{s}}} \quad f_{\min }=\Delta f=\frac{1}{t_{\mathrm{m}}}
$$

The data-processing part exists of a transfer function estimation based on the input and output time signals. After the measurement part, the input signal $X=T$ ('Torque' in figure 5) and the output signal $Y=\dot{\theta}$ ('Velocity' in figure 5) are saved. During the settling time to reach the desired position, the system is not in its linear operating point. This data is therefore deleted. The transfer function $H_{1}(s)$ is then estimated from the cross power spectral density $P_{Y X}$ of $X$ and $Y$, and the power spectral density $P_{X X}$ of $X$ ( [6], [8]):

$$
H_{1}(s)=\frac{P_{Y X}(s)}{P_{X X}(s)}
$$

For clarification, simulation results for the operating points listed in Table II are given with well-chosen and realistic settings listed in table III.

TABLE III

IDENTIFICATION SETTINGS

\begin{tabular}{|c|c|}
\hline Time signal & $t_{\mathrm{m}}=100 \mathrm{~s}, t_{\mathrm{s}}=0.0001 \mathrm{~s}$ \\
\hline Torque noise & $A=0.5 \mathrm{Nm}$ \\
\hline Position control & $K_{\mathrm{p}}=0.51 / \mathrm{s}$ \\
\hline Speed control & $K_{\mathrm{i}}=0.001 \mathrm{Nm} \cdot \mathrm{s} / \mathrm{rad}, T_{\mathrm{i}}=0.0036 \mathrm{~s}$ \\
\hline
\end{tabular}

The first experimental result is shown in figure 7 and is obtained at the position of high inertia $\theta_{\mathrm{a}}$. A clear correspondence is found between the estimated transfer function $H_{1}(s)$ and the linearised transfer function $G_{1}(s)$, which validates the proposed approach.

The simulated step response of the position $\theta$ in figure 8 

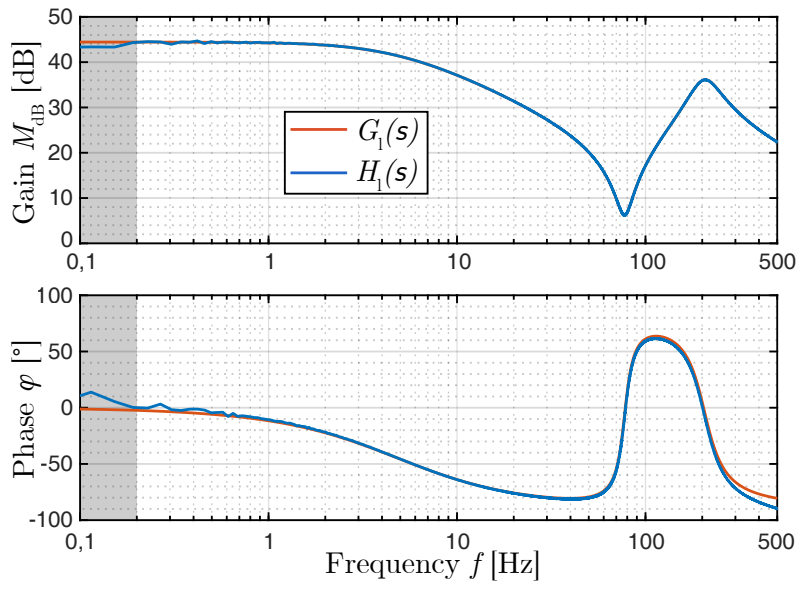

Fig. 7. Bode of $G_{1}(s)$ and $H_{1}(s)$ at high inertia $\theta_{\mathrm{a}}$

confirms that the identification settings in Table III are wellchosen. After transient behaviour during the settling time (marked in grey), the amplitude of the oscillations around the operating point $\theta^{*}$ is $\pm 8^{\circ}$ which agrees with the guideline of $2^{\circ}-10^{\circ}$. Note that the time axis is limited to $20 \mathrm{~s}$, while the measurement time is 100s. Figure 9 confirms that with these limited oscillations, the load torque $T_{1}\left(\theta^{*}\right)$ and load inertia $J_{1}\left(\theta^{*}\right)$ are respectively nearly equal to the constant values $T_{1}$ and $J_{1}$ in the operating point. As a result, the desired linear system behaviour is achieved.

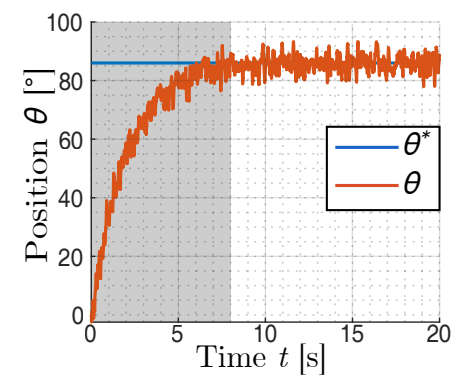

Fig. 8. Step response for $\theta^{*}=\theta_{\mathrm{a}}$
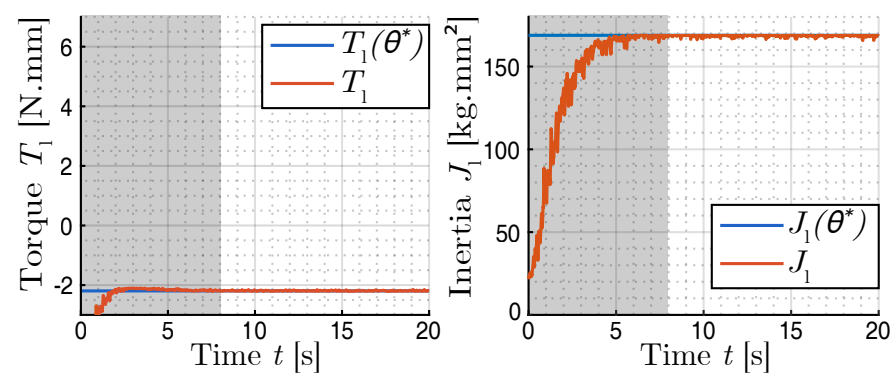

Fig. 9. Load torque $T_{1}$ and load inertia $J_{1}$ in the operating point $\theta^{*}=\theta_{\mathrm{a}}$

The second experimental result is shown in figure 10 and is obtained at the position of medium inertia $\theta_{\mathrm{b}}$. Again, a clear correspondence is found between $H_{1}(s)$ and $G_{1}(s)$. Yet, in the zone of antiresonance depicted in the close-up view, the correspondence is less accurate.
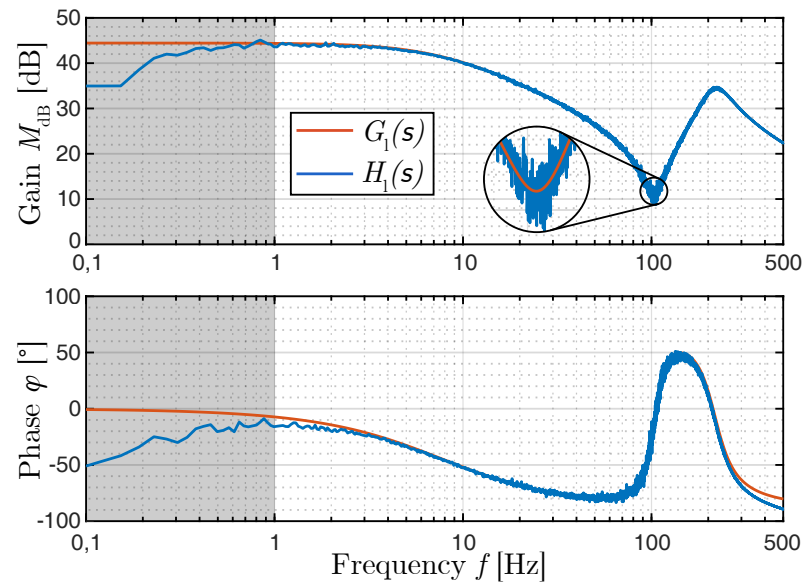

Fig. 10. Bode of $G_{1}(s)$ and $H_{1}(s)$ at medium inertia $\theta_{\mathrm{b}}$

This inaccuracy is explained by considering the load parameters in figure 11. For the same position oscillations of $\pm 8^{\circ}$, the load inertia $J_{1}$ oscillates a lot more around the value $J_{1}\left(\theta^{*}\right)$ in the operating point. This property is due to the
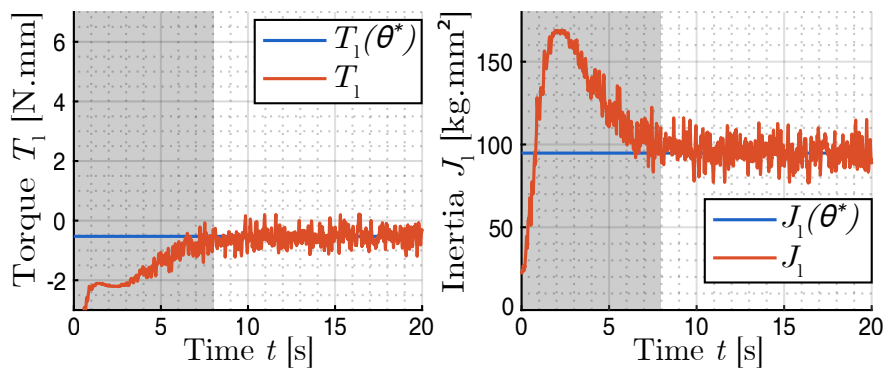

Fig. 11. Load torque $T_{1}$ and load inertia $J_{1}$ in the operating point $\theta^{*}=\theta_{\mathrm{b}}$

shape of the inertia profile. A close-up view of the grey region in the inertia profile in figure 3 is given in figure 12. For the same position variation $\Delta \theta$, the inertia variation $\Delta J_{1}$ at
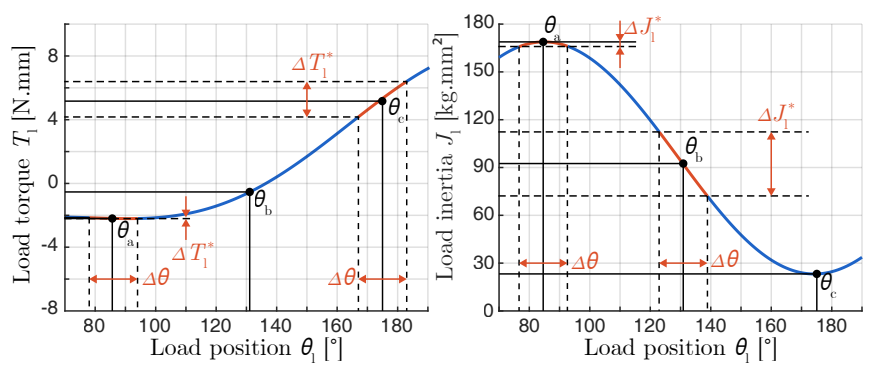

Fig. 12. Close-up view of the load parameters

operating point $\theta_{\mathrm{b}}$ is much larger than at operating point $\theta_{\mathrm{c}}$. Due to this large inertia variation, the desired linear system behaviour is not maintained resulting in a more inaccurate estimated transfer function $H_{1}(s)$.

The third result is shown in figure 13 and is obtained at the position of low inertia $\theta_{\mathrm{c}}$. Again, a clear correspondence is found between $H_{1}(s)$ and $G_{1}(s)$ except for the low frequency zone depicted in grey. In this zone, both the gain and phase 

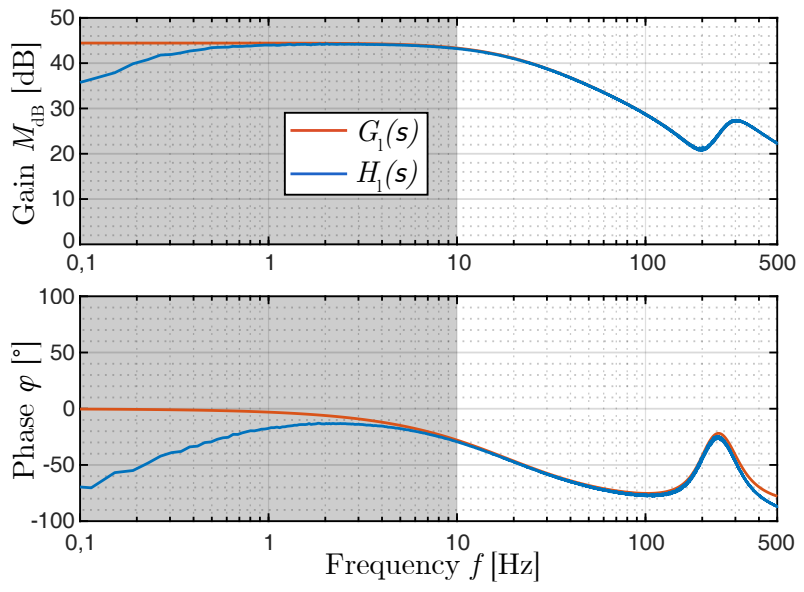

Fig. 13. Bode of $G_{1}(s)$ and $H_{1}(s)$ at low inertia $\theta_{\mathrm{c}}$

decrease.

This deviation with $G_{1}(s)$ is due to the shape of the load torque profile, shown in figure 12. For the same position variation $\Delta \theta$, the load torque variation $\Delta T_{1}$ at the operating point $\theta_{\mathrm{c}}$ is much larger than at the operating point $\theta_{\mathrm{a}}$. This results in large oscillations of the load torque $T_{1}$ around the value $T_{1}\left(\theta^{*}\right)$, as shown in figure 14 . Correspondingly, the linear system behaviour is not maintained and in this case results in a wrong estimated transfer function $H_{1}(s)$ at low frequency.
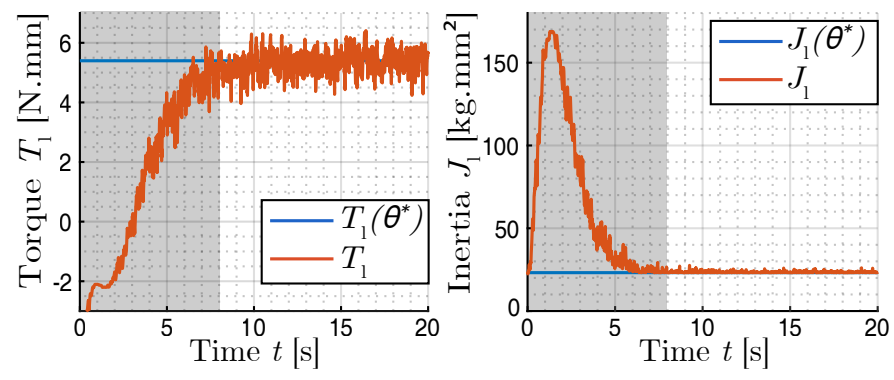

Fig. 14. Load torque $T_{1}$ and load inertia $J_{1}$ in the operating point $\theta^{*}=\theta_{\mathrm{c}}$

In conclusion, the proposed system identification approach is valid on the condition that the position-dependent mechanical parameters are nearly constant values during the measurement procedure. To do so, both the identification settings and the operating point must be well-chosen. The operating point is best chosen at a position where both the load torque and load inertia are in a local minimum or maximum, which is the case for the position of high inertia $\theta_{\mathrm{a}}$. In that point, the variation of these parameters is limited for a fixed position variation. This position variation is crucial for the identification procedure. If there are no oscillations in the position, there is no movement of the system. In other words there would be no frequency spectrum and obviously no frequency response to be identified. The amplitude of the position oscillations is a result of the chosen amplitude $A$ of the added torque noise. If this amplitude is chosen too high, the oscillations of the position are too high and the linear system behaviour is not maintained. Therefore the guideline of $2^{\circ}-10^{\circ}$ must be respected.

\section{MEASUREMENTS}

Figure 15 shows the identified open-loop system of the physical machine in figure 1 . The result is obtained with the proposed identification approach at the position $\theta_{\mathrm{c}}$ of low inertia. In the $20 \mathrm{~Hz}-200 \mathrm{~Hz}$ region a clear correspondence is found between the estimated transfer function $H_{1}(s)$ and the linearised transfer function $G_{1}(s)$.
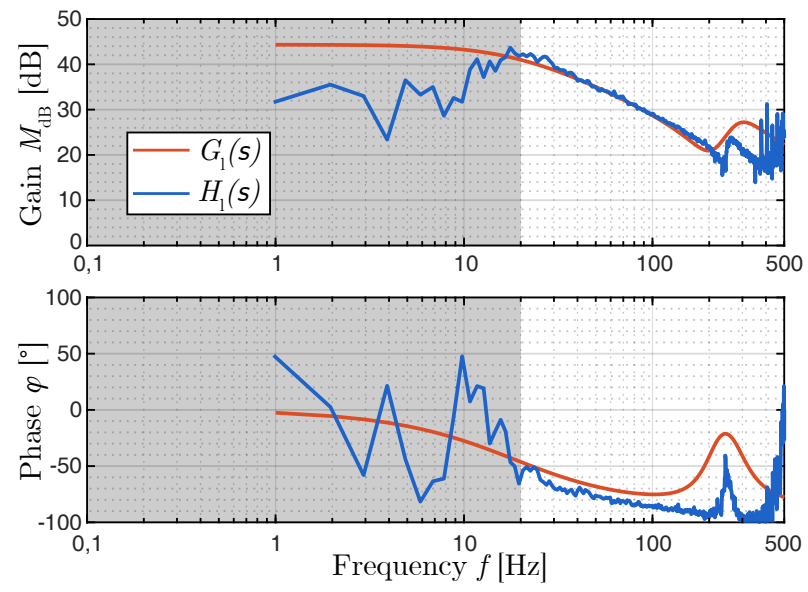

Fig. 15. Bode of $G_{1}(s)$ and $H_{1}(s)$ at low inertia $\theta_{\mathrm{c}}$

In the low-frequency zone, depicted in grey, the gain decreases. This property agrees with the simulated result in figure 13 and is due to the shape of the load torque profile (figure 12) in the operating point $\theta^{*}=\theta_{\mathrm{c}}$.

In the zone of antiresonance and resonance, both the gain and phase of $H_{1}(s)$ and $G_{1}(s)$ have the same shape but do not agree perfectly. This is due to a practical limitation of the SINAMICS drive software. The drive software is limited to a fixed number of samples $N$ that can be traced [12]. Due to this limit, the sample time $t_{s}$ and measurement time $t_{m}$ are not set according to the well-chosen identification settings in Table III, but at $t_{s}=0.001 \mathrm{~s}$ and $t_{m}=1 \mathrm{~s}$. According to (10) the maximum frequency $f_{\max }$ of the spectrum is than $500 \mathrm{~Hz}$ which is very close to the zone of antiresonance and resonance. Correspondingly, a low accurate estimation is done at this frequency. Despite this low measurement time $t_{m}$, the gain at the antiresonance frequency agrees quite well which validates that the proposed approach is useful for controller tuning.

An important remark is that although the simulation results in section IV suggest to select the operating point $\theta^{*}=\theta_{\mathrm{a}}$, the measurements are presented at $\theta^{*}=\theta_{\mathrm{c}}$. This is because at $\theta^{*}=\theta_{\mathrm{a}}$ the linear system behaviour of the physical machine is not maintained due to the presence of friction. The simplified two-mass system in figure 2 does not take friction into account and thus assumes that this non-linear property is negligible at $\theta^{*}=\theta_{\mathrm{a}}$. For the physical machine, friction is only negligible for a certain position range of the rotor which is illustrated with figure 16 . In position $\theta_{\mathrm{a}}$, the 
horizontal component of the action force $F_{\mathrm{r}}$ in the rod results in a friction force on the sliding $\mathrm{H}$-part. While in position $\theta_{\mathrm{c}}$, there is nearly no horizontal component, resulting in negligible friction. Note that, for convenience, only one $\mathrm{H}$ part and rod is shown in the figure.
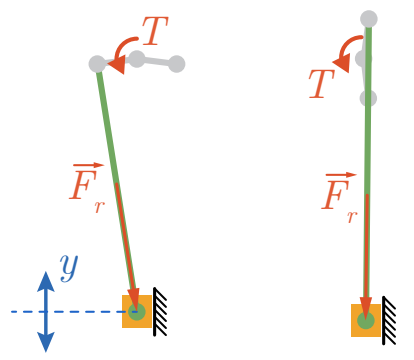

Fig. 16. Force $F_{\mathrm{r}}$ in a rod for the position $\theta_{\mathrm{a}}$ (left) and position $\theta_{\mathrm{c}}$ (right)

\section{CONCLUSION}

This paper presented an offline method for the identification of dynamic systems with position-dependent load properties. The feasibility of this method has been verified on both a physical machine and a simplified experimental model. Based on the results, the approach is valid and applicable for controller tuning. Moreover, the approach is proven to be implementable in standard commercial drives with low effort. However, an important condition is that the measurement is done at a well-selected rotor position.

\section{APPENDIX}

Figure 17 represents the load side of the two-mass system in figure 2. The load side is driven with a torque $T^{\prime}$ resulting in motion of the load inertia $J_{1}$. In order to achieve a virtual

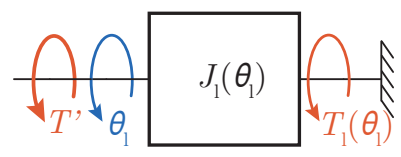

Fig. 17. Variable inertia system

change in position $d \theta_{\mathrm{l}}$, a virtual amount of work $d W$ must be carried out by the torque $T^{\prime}$. Due to the conservation of energy, this virtual change in work $d W$ results in a virtual change of total energy $d E$. The total energy equals the sum of kinetic energy $d E_{\mathrm{k}}$ and potential energy $d E_{\mathrm{p}}$.

$$
d W=d E_{\mathrm{p}}+d E_{\mathrm{k}}
$$

In this case, the change in kinetic energy is due to both a change of inertia $J_{1}$ and a change of angular velocity $\dot{\theta}$. The change in potential energy is due to a change of load torque $T_{1}$.

$$
\begin{gathered}
T^{\prime} d \theta_{\mathrm{l}}=d\left(\frac{1}{2} J_{\mathrm{l}}\left(\dot{\theta}_{\mathrm{l}}\right)^{2}\right)+T_{\mathrm{l}} d \theta_{\mathrm{l}} \\
\left(T^{\prime}-T_{1}\right) d \theta_{\mathrm{l}}=d\left(\frac{1}{2} J_{\mathrm{l}}\left(\dot{\theta}_{\mathrm{l}}\right)^{2}\right)
\end{gathered}
$$

Both members of (14) are divided by a virtual change in time $d t$ :

$$
\left(T^{\prime}-T_{1}\right) \frac{d \theta_{\mathrm{l}}}{d t}=\frac{d}{d t}\left(\frac{1}{2} J_{1}\left(\dot{\theta}_{\mathrm{l}}\right)^{2}\right)
$$

The time derivative of the right member in (15) can now be calculated. Note that the inertia $J_{1}$ is position-dependent and therefore time-dependent.

$$
\left(T^{\prime}-T_{1}\right) \dot{\theta}_{\mathrm{l}}=\frac{1}{2} \dot{J}_{1}\left(\dot{\theta}_{\mathrm{l}}\right)^{2}+J_{1} \dot{\theta}_{\mathrm{l}} \ddot{\theta}_{\mathrm{l}}
$$

Next, both members of (16) are divided by $\dot{\theta}_{\mathrm{l}}$ and the acceleration torque for a variable inertia system is found:

$$
T^{\prime}-T_{1}=\frac{1}{2} \dot{J}_{1} \dot{\theta}_{1}+J \ddot{\theta}_{1}
$$

In (17), the time-derivative of the inertia $\dot{J}_{1}$ is represented. An alternative is to write the equation with the positionderivative of the inertia included:

$$
\begin{array}{r}
\dot{J}_{\mathrm{l}}=\frac{d J_{1}}{d t}=\frac{d J_{1}}{d \theta_{\mathrm{l}}} \frac{d \theta_{1}}{d t} \\
T^{\prime}-T_{1}=\frac{1}{2} \frac{d J_{1}}{d \theta_{\mathrm{l}}}\left(\dot{\theta}_{\mathrm{l}}\right)^{2}+J \ddot{\theta}_{\mathrm{l}}
\end{array}
$$

\section{ACKNOWLEDGEMENT}

This work was financially supported by the Interreg 2 seas INCASE project (www.incase2seas.eu).

\section{REFERENCES}

[1] H. Dresig, F. Holzweibig, Dynamics of Machinery: Theory and Applications, Springer Science \& Business Media, New York, 2010.

[2] N. Van Oosterwyck, F. Vanbecelaere, M. Haemers, D. Ceulemans, K. Stockman, S. Derammelaere, CAD Enabled Trajectory Optimization and Accurate Motion Control for Repetitive Tasks ., 15th IEEE International Conference on Control and Automation.

[3] F. Roos, H. Johansson, J. Wikander, Optimal selection of motor and gearhead in mechatronic applications, Mechatronics 16 (1) (2006) 6372.

[4] N. Norman S., Design via frequency response, in: Control Systems Engineering, 6th Edition, Wiley, 2011, pp. 645-682.

[5] R. De Keyser, C. Ionescu, Frtool: A frequency response tool for cacsd in matlab, in: 2006 IEEE Conference on Computer Aided Control System Design, 2006 IEEE International Conference on Control Applications, 2006 IEEE International Symposium on Intelligent Control, 2006, pp. 2275-2280.

[6] S. Villwock, M. Pacas, Application of the Welch-Method for the Identification of Two- and Three-Mass-Systems (2008).

[7] S. E. Saarakkala, M. Hinkkanen, Identification of Two-Mass Mechanical Systems Using Torque Excitation: Design and Experimental Evaluation, IEEE Transactions on Industry Applications 51 (2015) 4180-4189.

[8] A. Wahrburg, E. Jelavic, S. Klose, K. D. Listmann, Robust SemiAutomatic Identification of Compliantly Coupled Two-Mass Systems, IFAC 50 (1) (2017) 14569-14574.

[9] BECKHOFF, TC3 Bode Plot Manual, BECKHOFF, 2017, p. 46.

[10] M. A. Valenzuela, J. M. Bentley, R. D. Lorenz, Evaluation of torsional oscillations in paper machine sections, IEEE Transactions on Industry Applications 41 (2005) 493-501.

[11] G. Berselli, F. Balugani, M. Pellicciari, M. Gadaleta, Energy-optimal motions for Servo-Systems: A comparison of spline interpolants and performance indexes using a CAD-based approach, ROBOTICS AND COMPUTER-INTEGRATED MANUFACTURING 40 (2016) 55-65.

[12] SIEMENS, SINAMICS S120 Drive Functions; Function manual, Tech. rep. (2014). 